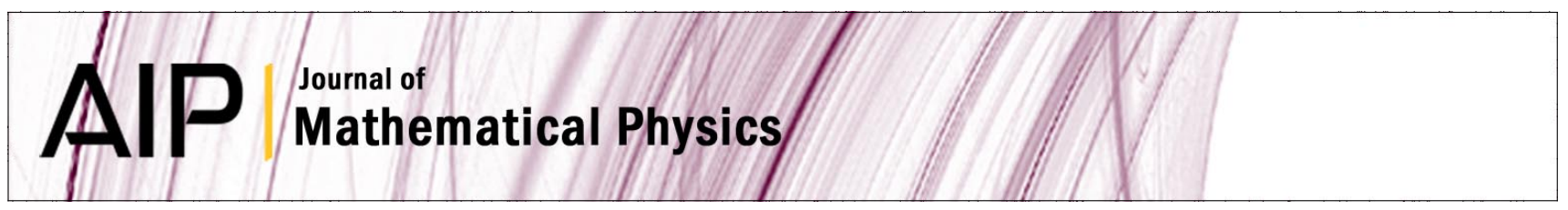

\title{
Information-theoretical derivation of an extended thermodynamical description of radiative systems
}

J. Fort and J. E. Llebot

Citation: J. Math. Phys. 39, 345 (1998); doi: 10.1063/1.532327

View online: http://dx.doi.org/10.1063/1.532327

View Table of Contents: http://jmp.aip.org/resource/1/JMAPAQ/v39/i1

Published by the American Institute of Physics.

\section{Additional information on J. Math. Phys.}

Journal Homepage: http://jmp.aip.org/

Journal Information: http://jmp.aip.org/about/about_the_journal

Top downloads: http://jmp.aip.org/features/most_downloaded

Information for Authors: http://jmp.aip.org/authors

\section{ADVERTISEMENT}

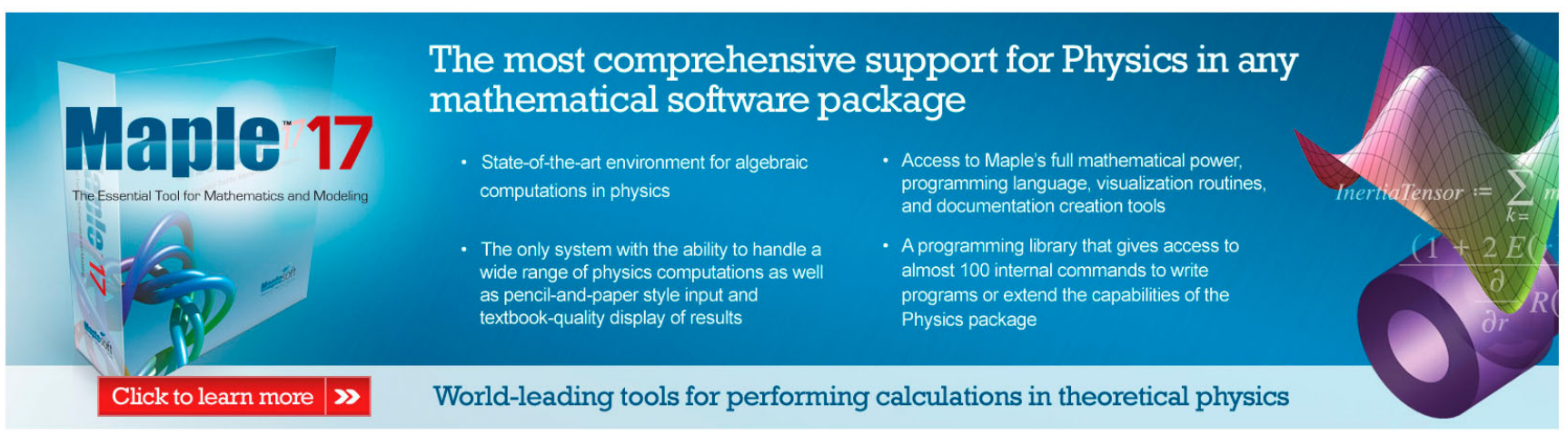




\section{Information-theoretical derivation of an extended thermodynamical description of radiative systems ${ }^{a)}$}

J. Fort ${ }^{\text {b) }}$

Secció de Fisica, Department d'Enginyeria Industrial, Escola Politècnica Superior, Universitat de Girona, Avda. Lluís Santaló s/n, 17071 Girona, Catalonia, Spain

J. E. Llebot

Grup de Física, Departament de Ciències Ambientals, Facultat de Ciències, Universitat de Girona, Plaça de l'Hospital 6, 17071 Girona, Catalonia, Spain

(Received 15 July 1997; accepted for publication 28 August 1997)

A radiative equation of the Cattaneo-Vernotte type is derived from information theory and the radiative transfer equation. The equation thus derived is a radiative analog of the equation that is used for the description of hyperbolic heat conduction. It is shown, without recourse to any phenomenological assumption, that radiative transfer may be included in a natural way in the framework of extended irreversible thermodynamics (EIT). (C) 1998 American Institute of Physics.

[S0022-2488(98)03001-1]

\section{INTRODUCTION}

Radiative transfer has recently become an active area of research within extended irreversible thermodynamics (EIT). However, most approaches to the subject have considered the description of purely radiation systems (photon gas) ${ }^{1-3}$ whereas a joint thermodynamical description of radiation and matter is certainly necessary. This conclusion can be reached as follows. Because photons do not interact among themselves, it is the interaction between radiation and matter that drives an isolated radiative system towards equilibrium. ${ }^{4}$ More generally, since in the absence of matter photons cross a small volume centered about any given point without any interaction, it is clear that the distribution function cannot be determined by the macroscopic properties at the point considered: instead, it will be determined by the properties of the emitting source. Similar arguments to the previous ones have led to the idea ${ }^{5,6}$ that it does not seem possible to reach valid thermodynamical conclusions with regards to radiative transfer on the basis of any picture that does not take into account the absorption and emission of radiation by matter. Some phenomenological models that include matter as well as radiation have been proposed (see, e.g., Refs. 7 and 8). However, a microscopic approach should be sought in order to see to what extent the hypotheses that lie at the ground of such phenomenological models can be justified. In this paper, we make use of information theory in order to present such a microscopic approach. It will be very useful, before doing so, to briefly recall two information-theoretical results in conductive, purely matter systems. For an ideal monatomic gas, maximization of the entropy density under the constraints of fixed molecule number density $n$, vanishing barycentric velocity, fixed internal energy density $\rho u_{m}$ ( $\rho$ stands for the matter density), and fixed conductive heat flux $\mathbf{q}$ leads, keeping terms up to second-order in $\mathbf{q}$, to the following results ${ }^{3}$ (see also Ref. 6) for the generalized Gibbs equation and for the temperature $\theta$ of extended irreversible thermodynamics

$$
d s_{m}=\frac{1}{\theta} d u_{m}-\frac{2 m}{5 n k_{B}^{2} T_{m}^{3} \rho} \mathbf{q} d \mathbf{q}
$$

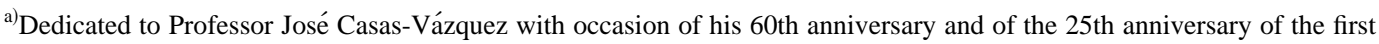
paper on Extended Irreversible Thermodynamics by his UAB group.

${ }^{b)}$ Electronic mail: jfort@songoku.udg.es
} 


$$
\frac{1}{\theta}=\frac{1}{T_{m}}\left(1+\frac{2 \rho}{5 n^{3} k_{B}{ }^{3} T_{m}{ }^{3}} \mathbf{q} \cdot \mathbf{q}\right),
$$

where $s_{m}$ is the specific entropy, $m$ is the molecular mass (so that $\rho=m n$ ), $k_{B}$ stands for the Boltzmann constant, $T_{m}$ is the kinetic local-equilibrium temperature, which satisfies that ${ }^{9,6}$

$$
u_{m}=3 k_{B} T_{m} / 2 m,
$$

and the definition of $\theta$ for the matter system under consideration is

$$
\theta^{-1} \equiv \frac{\partial s_{m}}{\partial u_{m}}
$$

Equations (1) and (2) have also been derived without making use of information theory but beginning with the postulates of extended irreversible thermodynamics and making use of the results of Grad's 13-moment kinetic theory method. ${ }^{9}$ This has provided a conceptually reasonable, sound framework for a thermodynamical description of heat conduction in further-away-fromequilibrium states than those corresponding to local-equilibrium thermodynamics. Our purpose is here to propose the information-theoretical basis of such a framework for radiative, instead of conductive, heat transfer. Therefore, in the following sections we do not consider a matter system under a conductive heat flux $\mathbf{q}$ but a radiation-matter system under a radiative heat flux, which we shall call $\mathbf{F}$.

\section{INFORMATION THEORY}

Assuming that the matter content of the system is a classical ideal monatomic gas, the entropy density of the system is ${ }^{4}$

$$
\rho s=\rho s_{m}+\rho s_{r}=-k_{B} \int_{R^{3}} \frac{d^{3} p_{m}}{(2 \pi \hbar)^{3}} f_{m} \ln f_{m}+2 k_{B} \int_{R^{3}} \frac{d^{3} p_{r}}{(2 \pi \hbar)^{3}}\left[\left(1+f_{r}\right) \ln \left(1+f_{r}\right)-f_{r} \ln f_{r}\right],
$$

where the subindexes $m$ and $r$ stand for matter and radiation (photons), and $s, f$, and $p$ are the corresponding entropies per unit mass, distribution functions, and momenta, respectively. Here $\hbar$ is the Planck constant $h$ divided by $2 \pi$. We have used the same notation as in Ref. 4: for example, $f_{m}$ stands for the number of molecules in $d^{3} p_{m}$ and $d^{3} x$, divided by $d^{3} p_{m} d^{3} x /(2 \pi \hbar)^{3}$, with $d^{3} x$ a differential of volume (in this way, $\ln f_{m}$ is dimensionless).

We will make use of the well-known information-theoretical approach ${ }^{10,11}$ by maximizing (5) under the macroscopic constraints of fixed total energy density $\rho u$, molecular number density $n$, and radiative energy flux $\mathbf{F}$ of the system,

$$
\begin{gathered}
\rho u=\rho u_{m}+\rho u_{r}=\int_{R^{3}} \frac{d^{3} p_{m}}{(2 \pi \hbar)^{3}} \frac{p_{m}^{2}}{2 m} f_{m}+2 \int_{R^{3}} \frac{d^{3} p_{r}}{(2 \pi \hbar)^{3}} p_{r} c f_{3}, \\
n=\int_{R^{3}} \frac{d^{3} p_{m}}{(2 \pi \hbar)^{3}} f_{m}, \\
\mathbf{F}=2 \int_{R^{3}} \frac{d^{3} p_{r}}{(2 \pi \hbar)^{3}} p_{r} c \mathbf{c} f_{r},
\end{gathered}
$$

where $u_{m}$ is the matter specific internal energy and $u_{r}$ is the radiation energy, also per unit mass of matter. In Eq. (6) we have assumed that the barycentric velocity of the matter gas vanishes (this 
allows us to concentrate on the topic that is of interest: we are neglecting heat convection in addition to heat conduction). In Eq. (8), $\mathbf{c}=c \hat{\Omega}$ is the velocity of a photon moving in a direction with unit vector $\hat{\Omega}$ and $c$ is the speed of light in vacuo. Maximization of (5) under the constraints (6)-(8) finally yields for the distribution functions

$$
\begin{gathered}
f_{m}=\exp \left[-1-\lambda-\beta \frac{p_{m}^{2}}{2 m}\right], \\
f_{r}=\frac{1}{\exp \left[\beta p_{r} c-\boldsymbol{\gamma} \cdot p_{r} c \mathbf{c}\right]-1},
\end{gathered}
$$

where $\lambda, \beta$, and $\gamma$ are Lagrange multipliers.

\section{EXTENDED IRREVERSIBLE THERMODYNAMICS OF RADIATIVE SYSTEMS}

We now follow the usual information-theoretical procedure ${ }^{9,12}$ in order to derive an extended Gibbs equation, which will be used to show that radiative transfer can be included in the framework of EIT. Since we have assumed the matter gas to be at rest, the equation of continuity implies that matter density does not change in time, i.e., the density differential vanishes, so that $d n=0$. From this fact and Eq. (5) it is not difficult to find out expressions for $d s_{m}$ and $d s_{r}$, in which we make use of (9) and (10), respectively. In this way and after use of (6)-(8) we arrive at $d s_{m}=k_{B} \beta d u_{m}$ and $d s_{r}=k_{B} \beta d u_{r}-\left(k_{B} / \rho\right) \boldsymbol{\gamma} \cdot d \mathbf{F}$. Therefore

$$
d s=d s_{m}+d s_{r}=k_{B} \beta d u-\frac{k_{B}}{\rho} \boldsymbol{\gamma} \cdot d \mathbf{F},
$$

where we note that the entropy per unit mass of the radiation-matter system under consideration is a function of the total (radiation and matter) specific energy $u$ and of the radiative heat flux $\mathbf{F}$. Because of this information-theoretical result, we propose the following generalization of the definition (4),

$$
\theta^{-1} \equiv \frac{\partial s}{\partial u}
$$

From Eqs. (12) and (11) we have

$$
\theta=\frac{1}{k_{B} \beta} \text {. }
$$

On the other hand, it will be useful to follow Mihalas and Mihalas ${ }^{13}$ by introducing a radiative parameter $T_{r}$ through the following equation;

$$
\rho u_{r}=a T_{r}^{4}
$$

with $a=\pi^{2} k_{B}^{4} / 15 c^{3} \hbar^{3}$ the blackbody constant.

After substitution of the matter distribution function (9) into (7) and into $\rho u_{m}$, given by (6), and integration over all possible values of $\mathbf{p}_{m}$, it is easy to reach the result $\rho u_{m}=3 n / 2 \beta$, which together with (3) implies that $T_{m}=1 / k_{B} \beta$. Thus we have, taking into account Eq. (13),

$$
\theta=T_{m},
$$

and this result is consistent with Eq. (2), because we are not dealing with conductive but with radiative heat transfer (i.e., we have $\mathbf{q}=0$ although $\mathbf{F} \neq 0$ in general). 
We now look for a relationship between $\theta$ and $T_{r}$. We make use of the radiation distribution function (10) into (8) and into $\rho u_{r}$, given by (6). Since both integrations over $\mathbf{p}_{r}$ are rather complicated, we assume that $\gamma=(0,0, \gamma)$, integrate with the use of formula 3.411-1 of Ref. 14 and obtain

$$
\begin{gathered}
\rho u_{r}=\frac{a}{k_{B}{ }^{4} \beta^{4}} \frac{1+\epsilon^{2} / 3}{\left(1-\epsilon^{2}\right)^{3}}, \\
\mathbf{F}=\frac{4 c a}{3 k_{B}{ }^{4} \beta^{4}} \frac{\boldsymbol{\epsilon}}{\left(1-\epsilon^{2}\right)^{3}} \equiv(0,0, F),
\end{gathered}
$$

with

$$
\boldsymbol{\epsilon} \equiv \frac{c}{\beta} \gamma \equiv(0,0, \epsilon)
$$

From Eqs. (14), (16), and (13) we obtain

$$
\theta=T_{r}\left(\frac{\left(1-\epsilon^{2}\right)^{3}}{1+\epsilon^{2} / 3}\right)^{1 / 4}
$$

This equation relates $\theta$ to $T_{r}$, but $\epsilon$ should be written in terms of macroscopic observables if we want the radiative theory to be put at the same level as the conductive one: according to the conductive relationship (2), for a given value of $m$ (i.e., for a given matter gas) $\theta$ is a known function of $T_{m}$ if the values of the macroscopic observables $\rho$ and $\mathbf{q}$ are known. We can cope with this problem by noting that (16), (17), and (14) can be combined to yield

$$
F=4 c a T_{r}^{4} \frac{\epsilon}{3+\epsilon^{2}},
$$

from which it follows that

$$
\epsilon=\frac{2-\sqrt{4-3 F^{2} / c^{2} a^{2} T_{r}^{8}}}{F / c a T_{r}^{4}} .
$$

We make use of (20) in the denominator in (19) and then apply Eq. (21). Since we will here be interested in near-equilibrium results, we assume that the value of $F$ is low enough so that $\theta$ can be approximated by its MacLaurin expansion up to second order in $F$. This yields

$$
\frac{1}{\theta}=\frac{1}{T_{r}}\left(1+\frac{15}{32 c^{2} a^{2} T_{r}{ }^{8}} F^{2}\right),
$$

and this equation, which applies to radiative transfer, is analogous to the conductive equation (2). The second-order result (22) is in agreement with the expression for $\theta$ that has been obtained [Eqs. (27) and (17) in Ref. 3] by partial derivation of the radiative entropy density (which was derived on phenomenological grounds in Refs. 1 and 15) with respect to the radiative energy density. Equation (22) has also been previously derived by Casas-Vázquez and Jou for a purely radiation system [see Eq. (30) in Ref. 16], by making use of a fluctuation theory result and of the EIT phenomenology. However, there is a very important difference between these approaches and the information-theoretical one presented here: since in our definition (12) for $\theta$ we have made use of the total radiation and matter entropy and energy densities, the conceptual objections presented in 
the first lines in this letter do not apply to our analysis. A generalized Gibbs equation can be finally derived from (11), (18), (13), and (21). Keeping in mind that we are interested in the second-order approximation for $\theta$ and $s$, we obtain

$$
d s=\frac{1}{\theta} d u-\frac{3}{4 c^{2} a \rho T_{r}^{5}} \mathbf{F} \cdot d \mathbf{F},
$$

which is analogous to the conductive result (1). On the other hand, we note from (3), (15), and (22) that $u_{m}$ is a function of $T_{r}$ [or $u_{r}$, see Eq. (14)] and $\mathbf{F}$. Therefore, although in Eq. (23) we have taken the total specific energy $u=u_{m}+u_{r}$ and $\mathbf{F}$ as independent variables, it is clear that in this model $u_{r}$ and $\mathbf{F}$ may also be chosen as independent variables. It is easy to relate $d u_{m}$ to $d u_{r}$ and $d \mathbf{F}$. Making use of the relationship thus obtained and of (23), applying (22), and neglecting higher-order terms, one finally obtains the extended Gibbs equation in the new variables,

$$
d s=\left(\frac{1}{\theta}\left\{1+\frac{3 k_{B} \rho^{1 / 4}}{8 m a^{1 / 4} u_{r}^{3 / 4}}\right\}+\frac{315 k_{B} F^{2}}{256 m \rho^{2} c^{2} u_{r}^{3}}\right) d u_{r}-\left(\frac{45 k_{B}}{32 m c^{2} \rho^{2} u_{r}^{2}}+\frac{3 a^{1 / 4}}{4 c^{2} \rho^{9 / 4} u_{r}^{3 / 4}}\right) \mathbf{F} \cdot d \mathbf{F} .
$$

From this equation, (22), and (14), it is straightforward to check that $d s$ is an exact differential, i.e.,

$$
\frac{\partial^{2} s}{\partial \mathbf{F} \partial u_{r}}=\frac{\partial^{2} s}{\partial u_{r} \partial \mathbf{F}}
$$

so that the total (i.e., radiation and matter) specific entropy of the system is a state function, as it should.

\section{APPLICATION}

Kremer and Müller ${ }^{1}$ were able to deduce a radiative equation which is essentially of the Cattaneo-Vernotte relaxational type [their second Eq. (7.1)], but the corresponding relaxation time and radiative conductivity were not found out. We will now provide a microscopic derivation of that relaxational equation and find out the relaxation time and radiative conductivity explicitly.

Let us mention, for the sake of completeness, that making use of Eq. (7) it is not difficult to show that the matter distribution function (9) is locally Maxwellian, with temperature $T_{m}=\theta$ [see Eq. (15)]. On the other hand, making use of Eqs. (18), (21), (13), and (22), the informationtheoretical radiative distribution function (10) reads, up to second order in $\mathbf{F}$,

$$
f_{r}=f_{r}^{(0)}\left(1+\phi^{(1)}+\phi^{(2)}+\cdots\right),
$$

with

$$
\begin{gathered}
f_{r}^{(0)}=\frac{1}{e^{x}-1}, \\
\phi^{(1)}=\frac{3 x}{4} \frac{e^{x}}{e^{x}-1} \hat{\Omega} \cdot \mathbf{f}, \\
\phi^{(2)}=\widetilde{\phi}^{(2)}+\tilde{\tilde{\phi}}^{(2)}, \\
\widetilde{\phi}^{(2)}=-\frac{15 x}{32} \frac{e^{x}}{e^{x}-1} \mathbf{f} \cdot \mathbf{f},
\end{gathered}
$$




$$
\tilde{\tilde{\phi}}^{(2)}=\frac{9 x^{2}}{32} \frac{e^{x}\left(e^{x}+1\right)}{\left(e^{x}-1\right)^{2}}(\hat{\Omega} \cdot \mathbf{f})^{2},
$$

where we have defined the following dimensionless quantities:

$$
\mathbf{x} \equiv \frac{c}{k_{B} T_{r}} \mathbf{p}_{r}, \quad x \equiv|\mathbf{x}|, \quad \hat{\Omega} \equiv \frac{\mathbf{c}}{c}=\frac{\mathbf{p}_{r}}{p_{r}}=\frac{\mathbf{x}}{x}, \quad \mathbf{f} \equiv \frac{\mathbf{F}}{c a T_{r}^{4}}=\frac{\mathbf{F}}{c u_{r} \rho},
$$

f being the reduced flux. We have written (27)-(31) in terms of $T_{r}$, rather than in terms of $\theta=T_{m}$, for later convenience. The distribution function (26)-(31) is a radiative analog of the DominguezJou distribution function [Eqs. (30), (33), and (34) in Ref. 3], which was also derived making use of information theory but applies to a nonrelativistic classical monatomic ideal gas under a conductive heat flux. Both in radiative and conductive systems (present paper and Ref. 3, respectively), information theory provides a simple derivation for the second-order non-equilibrium correction $\phi^{(2)}$. This correction is very difficult to find out in the framework of the kinetic theory of gases ${ }^{17,18}$ of conductive, purely matter systems. It is worthwhile to mention that information theory is very simple mathematically but has the disadvantage that the question of what constraints should be imposed in maximizing the entropy density is an open one at present. On the other hand, it has been stressed ${ }^{5}$ that a well-established phenomenological thermodynamics of radiative transfer does not seem to be available. This is in contrast with the situation in purely matter systems, where phenomenological thermodynamics may be a useful guide in the choice of the informationtheoretical constraints (see Refs. 6, 12, 19, and, in connection with this, pages 46-47 in Ref. 9).

Our purpose here is to show that a relaxational equation follows from the radiative distribution function. In order to do so, we shall first show that although both (22) and (23) on one hand, and (26) -(31) on the other, have been obtained working up to second order in $\mathbf{F}$, only the first nonequilibrium correction $\phi^{(1)}$ contributes to the second-order entropy corresponding to (22) and (23). Making use of Eq. (26) and of the MacLaurin expansion

$$
\ln \left(a_{0}+a_{1} F+a_{2} F^{2}+\cdots\right)=\ln a_{0}+\frac{a_{1}}{a_{0}} F+\left(\frac{2 a_{2}}{a_{0}}-\frac{a_{1}^{2}}{a_{0}^{2}}\right) \frac{F^{2}}{2}+O\left(F^{3}\right)
$$

into the expression for $\rho s_{r}$, given by Eq. (1), we obtain, up to second order,

$$
\begin{aligned}
\rho s_{r}= & \rho s_{r}^{(0)}+2 k_{B} \int_{R^{3}} \frac{d^{3} p_{r}}{(2 \pi \hbar)^{3}} f_{r}^{(0)} \ln \left(\frac{1}{f_{r}^{(0)}}+1\right) \phi^{(1)} \\
& -2 k_{B} \int_{R^{3}} \frac{d^{3} p_{r}}{(2 \pi \hbar)^{3}} f_{r}^{(0)} \ln \left(\frac{1}{f_{r}^{(0)}}+1\right) \phi^{(2)} \\
& -k_{B} \int_{R^{3}} \frac{d^{3} p_{r}}{(2 \pi \hbar)^{3}} \frac{f_{r}^{(0)}}{1+f_{r}^{(0)}} \phi^{(1)^{2}},
\end{aligned}
$$

with

$$
\rho s_{r}^{(0)}=2 k_{B} \int_{R^{3}} \frac{d^{3} p_{r}}{(2 \pi \hbar)^{3}}\left[\left(1+f_{r}^{(0)}\right) \ln \left(1+f_{r}^{(0)}\right)-f_{r}^{(0)} \ln f_{r}^{(0)}\right] .
$$

The second term on the right-hand side of (33) vanishes because (27) and (28) imply that the corresponding integrand changes sign under the transformation $\mathbf{p}_{r} \rightarrow-\mathbf{p}_{r}$. The third term, which contains $\phi^{(2)}$, is also seen to vanish after substitution of (27) and (29)-(31), application of the integral theorem (which is easy to prove) 


$$
\int_{R^{3}} d^{3} x F(x)(\mathbf{x} \cdot \mathbf{g})^{2}=\frac{|\mathbf{g}|^{2}}{3} \int_{R^{3}} d^{3} x x^{2} F(x)
$$

[with $\mathbf{g}$ any vector and $F(x)$ an arbitrary function], and use of the integrals

$$
\int_{0}^{\infty} d x \frac{x^{4} e^{x}}{\left(e^{x}-1\right)^{2}}=\frac{4 \pi^{4}}{15}, \quad \int_{0}^{\infty} d x \frac{x^{5} e^{2 x}}{\left(e^{x}-1\right)^{3}}=\frac{2 \pi^{4}}{3}+60 \zeta(5)
$$

and

$$
\int_{0}^{\infty} d x \frac{x^{5} e^{x}}{\left(e^{x}-1\right)^{3}}=\frac{2 \pi^{4}}{3}-60 \zeta(5)
$$

$\zeta(z)$ being the Riemann zeta function. The vanishing of the second and third terms on the right-hand side of the radiative entropy density (33) can be regarded as the radiative analogue of the (e.g., kinetic-theoretical) result ${ }^{17,9}$ for purely matter systems

$$
\int_{R^{3}} \frac{d^{3} p_{m}}{(2 \pi \hbar)^{3}} f_{m}^{(0)} \phi_{m}^{(k)}=0
$$

with $f_{m}^{(0)}$ the Maxwell-Boltzmann distribution function and $\phi_{m}^{(k)}$ the $k$ th-order correction due to heat conduction and/or convection: this leads to ${ }^{9}$

$$
\rho s_{m}=\rho s_{m}^{(0)}-\frac{k_{B}}{2} \int_{R^{3}} \frac{d^{3} p_{m}}{(2 \pi \hbar)^{3}} f_{m}^{(0)} \phi_{m}^{(1)^{2}},
$$

so that $\phi_{m}^{(2)}$ does not contribute to the second-order approximation to the matter entropy density in conductive and/or convective situations, just as (33) becomes

$$
\rho s_{r}=\rho s_{r}^{(0)}-k_{B} \int_{R^{3}} \frac{d^{3} p_{r}}{(2 \pi \hbar)^{3}} \frac{f_{r}^{(0)}}{1+f_{r}^{(0)}} \phi^{(1)^{2}},
$$

so that $\phi^{(2)}$ does not contribute to the second-order radiation entropy density in radiative situations. We mention that this conclusion can also be reached, after rather tedious calculations, by checking that integration of the first and fourth terms on the right-hand side of (33) yields the same result for $\rho s_{r}$ as that obtained by substitution of (10) into $\rho s_{r}$, given by (5), integration, and expansion of the result up to second order in $\mathbf{F}$. By following either of the procedures it is obtained that

$$
\rho s_{r}=\rho s_{r}^{(0)}-\frac{3}{8 a c^{2} T_{r}^{5}} F^{2}+O\left(F^{3}\right)
$$

with $\rho s_{r}^{(0)}=4 a T_{r}^{3} / 3$.

In the special case of thermodynamic equilibrium $(\mathbf{F}=0)$, Eq. (21) becomes $\epsilon=0$, (15) and (19) yield $\theta=T_{m}=T_{r}$, and (26)-(31) reduce to the Planck distribution function, $f_{r}=f_{r}^{(0)}$, as they should. In the same case, (34) becomes the usual expression for the radiation entropy density in equilibrium, i.e., ${ }^{4} \rho s_{r}=\rho s_{r}^{(0)}=4 a T_{r}^{3} / 3$, and we have, instead of (23), that $d s=\left(1 / T_{r}\right) d u$. This is a Gibbs equation. Therefore, analogously to what is done in phenomenological thermodynamics of matter systems, ${ }^{9} T_{r}$ may be called the local-equilibrium temperature and $s_{r}^{(0)} \equiv s_{r l e}$ may be called the local-equilibrium radiative entropy. 
We now turn our attention to the second-order theory. In order to verify its consistency, it is rather important to note that Eq. (34) can also be obtained very easily (although without finding $\rho s_{r}^{(0)}$ explicitly) from (23), (22), and the radiative part of the local Gibbs equation, i.e., $d s_{r l e}$ $=\left(1 / T_{r}\right) d u_{r}$, in the following way. From $d s_{m}=(1 / \theta) d u_{m}$ [see the text above Eq. (11) and Eq. (13)] and (23) we have $d s_{r}=(1 / \theta) d u_{r}-\left(3 / 4 c^{2} a \rho T_{r}^{5}\right) \mathbf{F} \cdot d \mathbf{F}$. By integration with the use of (22) and (14) we find the second-order result $s_{r}=s_{r l e}-\left(3 / 8 a c^{2} \rho T_{r}^{5}\right) F^{2}$, in agreement with Eq. (34).

The fact, derived above Eq. (34), that $\phi^{(1)}$ contributes to the second-order radiation specific entropy, whereas $\phi^{(2)}$ does not, implies that the second-order thermodynamical results (22) and (23) correspond to the first-order distribution function, i.e., $f_{r} \approx f_{r}^{(0)}\left(1+\phi^{(1)}\right)$ (we stress that the same happens in EIT of purely matter systems, see pp. 83-87 in Ref. 9). From this result, we will finally be able to find an evolution equation.

It is well known that information theory on its own cannot yield evolution equations, simply because all it yields is an expression for the distribution function and an (extended) Gibbs equation. However, the information-theoretical distribution function can be ${ }^{20}$ and has in fact been combined with dynamical methods, such as the Boltzmann equation, ${ }^{21}$ the Liouville equation, ${ }^{22}$ the statistical operator method, ${ }^{23}$ or the radiative transfer equation. ${ }^{24}$ As it is well known, ${ }^{25,8}$, multiplication of the radiative transfer equation by $\hat{\Omega}$ and integration yields, under the grey approximation,

$$
\frac{1}{c} \frac{\partial \mathbf{F}}{\partial t}+c \boldsymbol{\nabla} \cdot \mathbf{P}_{r}=-\sigma_{a} \mathbf{F}
$$

with $\sigma_{a}$ the absorption coefficient, $\left[\boldsymbol{\nabla} \cdot \mathbf{P}_{r}\right]_{i} \equiv \Sigma_{k=1}^{3} \partial P_{r k i} / \partial x_{k}$ and $P_{r k i}$ the components of the pressure tensor of radiation, namely,

$$
P_{r k i}=\frac{2}{c} \int_{R^{3}} \frac{d^{3} p_{r}}{(2 \pi \hbar)^{3}} p_{r} c_{k} c_{i} f_{r} .
$$

Substitution of $f_{r} \approx f_{r}^{(0)}\left(1+\phi^{(1)}\right)$ in this expression and use of Eqs. (27), (28), and (32) shows that $\phi^{(1)}$ does not contribute to the pressure tensor. Use of formula (3.411.1) in Ref. 14 allows us to perform the remaining integration. This yields

$$
P_{r k i}=\frac{a}{3} T_{r}^{4} \delta_{k i}
$$

with $\delta_{k i}=1$ for $k=i$ and $\delta_{k i}=0$ for $k \neq i$. Use of (37) in Eq. (35) yields

$$
\tau \frac{\partial \mathbf{F}}{\partial t}+\mathbf{F}=-\lambda \boldsymbol{\nabla} T_{r}
$$

with

$$
\tau=\frac{1}{c \sigma_{a}}, \quad \lambda=\frac{4 c a}{3 \sigma_{a}} T_{r}^{3}
$$

Equation (38) is in agreement with a result previously derived in Ref. 1, but here it has been possible to introduce $\boldsymbol{\nabla} T_{r}$ instead of the gradient of the radiative energy density, and also to find out the explicit expressions (39) for the radiative relaxation time $\tau$ and the radiative conductivity $\lambda$. It is encouraging that in steady states Eqs. (38) and (39) become $\mathbf{F}=-\left(4 c a / 3 \sigma_{a}\right) T_{r}^{3} \boldsymbol{\nabla} T_{r}$, and this Fourier-type equation has been used since many years $\mathrm{ago}^{26}$ in the theory of stellar interiors. We have seen that information theory provides a statistical-mechanical derivation of the more general results (38) and (39). Similar, less rigorous expressions have been recently advanced on 
phenomenological grounds. ${ }^{8}$ Here, information theory has also allowed us to distinguish and relate $T_{m}$ to $T_{r}$ [defined by Eqs. (3) and (14), respectively] through (22) and (15), i.e.,

$$
\frac{1}{T_{m}}=\frac{1}{T_{r}}\left(1+\frac{15}{32 c^{2} a^{2} T_{r}^{8}} F^{2}\right) .
$$

We stress that this result is valid under the same conditions as those under which (38) and (39) hold.

From the generalized Gibbs equation (23), the energy balance law, namely ${ }^{27} \rho \partial u / \partial t=\boldsymbol{\nabla} \cdot \mathbf{F}$, and Eqs. (38) and (39) it is not difficult to follow the usual procedure ${ }^{9}$ and identify the entropy production rate, for low enough values of the flux $\mathbf{F}$, as

$$
\sigma^{s}=\frac{3 \sigma_{a}}{4 c a T_{r}^{5}} \mathbf{F} \cdot \mathbf{F},
$$

which is semi-positive definite, in agreement with the second law. According to (39) and (40), we may write $\sigma^{s}=\left(1 / \lambda T_{r}^{2}\right) \mathbf{F} \cdot \mathbf{F}$, which is analogous to the conductive result $\sigma^{s}=\left(1 / \lambda^{\prime} T_{m}^{2}\right) \mathbf{q} \cdot \mathbf{q}$, with $\lambda^{\prime}$ the thermal conductivity. Equation (40) shows that in the absence of matter $\left(\sigma_{a}=0\right)$ there is no entropy production. This corresponds to the fact that photons do not interact among themselves. Because radiative transfer in the vacuum in absolutely nonlocal, it has been previously argued ${ }^{6,5}$ that the entropy production must arise from the interaction of radiation with matter, although the explicit formula (40) does not seem to have been derived or proposed before.

We may also note that Eq. (38) is of the Cattaneo-Vemotte type: because of conceptual motivations (see, e.g., Ref. 9), and also because Fourier's law of heat conduction, i.e., q $=-\lambda^{\prime} \boldsymbol{\nabla} T_{m}$, is known to fail in very fast processes, ${ }^{28}$ a more general law, similar to (38), for heat conduction (instead of heat radiation), namely

$$
\tau^{\prime} \frac{\partial \mathbf{q}}{\partial t}+\mathbf{q}=-\lambda^{\prime} \boldsymbol{\nabla} T_{m}
$$

with $\tau^{\prime}$ the conductive relaxation time, has been considered since long ago. ${ }^{29,30}$ Equation (41) was a fundamental starting point ${ }^{31}$ for the development of the theory presently called extended irreversible thermodynamics (see, e.g., Refs. 9 and 20). The similarity between Eqs. (41) and (38), in itself, indicates the reasonability of including radiative transfer in the framework of EIT.

Still from another point of view, we may compare our results with previous proposals (see, e.g., Ref. 7) by saying that it seems now possible to approach the irreversible thermodynamics of radiative transfer without need, in principle, to make use of a temperature field that depends on frequency and direction. This is analogous to the fact that in nonequilibrium thermodynamics of heat conduction and convection ${ }^{17,9}$ there is no need to introduce a temperature field that depends on the molecular speed (or energy) and direction of motion.

Just to summarize, information theory provides a nonequilibrium extension of Planck's distribution function [Eqs. (26)-(31)], a microscopic derivation of a radiative equation of the Cattaneo-Vemotte type [Eqs. (38) and (39)], and it also leads to the conclusion that the range of nonequilibrium phenomena for which EIT provides an adequate framework is enlarged with the inclusion of radiative transfer.

\section{ACKNOWLEDGMENTS}

The authors would like to thank Professor D. Jou for stimulating discussions and Professor A. S. Cukrowski for providing us with the integral theorem used under Eq. (33) and with Ref. 18. This work has been partially funded by the Comisión Interministerial de Ciencia y Tecnología of the Spanish government under grant No. CL195-1867. 
${ }^{1}$ G. M. Kremer and I. Müller, J. Math. Phys. 33, 2265 (1992).

${ }^{2}$ M. Ferrer and D. Jou, Am. J. Phys. 63, 237 (1995), see especially the Appendix.

${ }^{3}$ R. Dominguez and D. Jou, Phys. Rev. E 51, 158 (1995).

${ }^{4}$ L. D. Landau and E. M. Lifshitz, Statistical Physics. Part I (Pergamon, Oxford, 1980).

${ }^{5}$ C. Essex, Adv. Thermodyn. 3, 435 (1990).

${ }^{6}$ R. E. Nettleton, Phys. Rev. E 53, 1241 (1996).

${ }^{7}$ H. F. Albert, J. Non-Equilib. Thermodyn. 16, 303 (1991).

${ }^{8}$ J. Fort and J. E. Llebot, J. Phys. A 29, 3427 (1996).

${ }^{9}$ D. Jou, J. Casas-Vázquez, and G. Lebon, Extended Irreversible Thermodynamics (Springer, Berlin, 1996).

${ }^{10}$ E. T. Jaynes, Phys. Rev. 106, 620 (1957); 108, 171 (1957); E. T. Jaynes: Papers on Probability, Statistics and Statistical Physics, edited by R. D. Rosenkrantz (Reidel, Dordrecht, 1983).

${ }^{11}$ F. Schlögl, Phys. Rep. 62, 267 (1980).

${ }^{12}$ R. E. Nettleton, J. Chem. Phys. 93, 8247 (1990).

${ }^{13}$ D. Mihalas and B. W. Mihalas, Foundations of Radiation Hydrodynamics (Oxford U.P., Oxford, 1984).

${ }^{14}$ I. S. Gradshteyn and I. M. Ryzhik, Table of Integrals, Series and Products (Academic, New York, 1984).

${ }^{15}$ A. M. Anile, S. Pennisi, and M. Sammartino, J. Math. Phys. 32, 544 (1991).

${ }^{16}$ J. Casas-Vázquez and D. Jou, Phys. Rev. E 49, 1040 (1994).

${ }^{17}$ S. Chapman and T. G. Cowling, The Mathematical Theory of Non-Uniform Gases (Cambridge U.P., Cambridge, 1970).

${ }^{18}$ M. S. Shavalieff, Prikl. Mat. Mekh. 42, 656 (1978) (in Russian).

${ }^{19}$ R. E. Nettleton, Phys. Rev. A 42, 4622 (1990).

${ }^{20}$ R. E. Nettleton and S. Sobolev, J. Non-Equilib. Thermodyn. 20, 205, 297 (1995); 21, 1 (1996).

${ }^{21}$ J. Fort and A. S. Cukrowski, Chem. Phys. 222, 59 (1997).

${ }^{22}$ B. Robertson, Phys. Rev. 144, 15 (1966); 160, 175 (1967).

${ }^{23}$ A. R. Vasconcellos, R. Luzzi, and L. S. Garcia-Colín, Phys. Rev. A 43, 6622, 6633 (1991).

${ }^{24}$ J. Fort, Physica A 243, 275 (1997).

${ }^{25}$ G. C. Pomraning, J. Quant. Spectrosc. Radiat. Transf. 27, 517 (1982).

${ }^{26}$ A. S. Eddington, The Internal Constitution of the Stars (Cambridge U.P., Cambridge, 1988), p. 101.

${ }^{27}$ S. R. De Groot and P. Mazur, Non-Equilibrium Thermodynamics (Dover, New York, 1984).

${ }^{28}$ See, e.g., D. D. Joseph and L. Preziosi, Rev. Mod. Phys. 61, 41 (1989); 62, 375 (1990).

${ }^{29}$ C. Cattaneo, Atti Sem. Mat. Fis. Univ. Modena 3, 33 (1948).

${ }^{30}$ P. Vernotte, C. R. Acad. Sci. Paris 246, 3154 (1958).

${ }^{31}$ R. E. Nettleton, Phys. Fluids 3, 216 (1960). 